\title{
PRKACA Gene Mutation
}

National Cancer Institute

\section{Source}

National Cancer Institute. PRKACA Gene Mutation. NCI Thesaurus. Code C156938.

A change in the nucleotide sequence of the PRKACA gene. 